\title{
Contribuições da análise do comportamento na escola: experiências do Programa de Pós-Graduação Stricto Sensu em Psicologia (PSSP) da PUC Goiás
}

Sonia Maria Mello Neves. Pontifícia Universidade Católica de Goiás Fábio Henrique Baia. Universidade de São Paulo. Universidade de Rio Verde Antônio Carlos Godinho Santos. Pontifícia Universidade Católica de Goiás Gabriela Sebastiana Ciriaco. Pontifícia Universidade Católica de Goiás Lorismario Ernesto Simonassi. Pontifícia Universidade Católica de Goiás Nagi Hanna Salm Costa. Pontifícia Universidade Católica de Goiás Rogério Guaita dos Santos Baia. Universidade de Rio Verde

\section{Resumo}

O Programa de Pós-Graduação Stricto Sensu em Psicologia (PSSP) da Pontifícia Universidade Católica de Goiás foi autorizado em 1999. A Análise do Comportamento é uma das áreas de concentração do programa. Ao longo desses 20 anos foram produzidos 115 teses e dissertações com embasamento behaviorista radical e cerca de 13\% dos trabalhos foram conduzidos em ambiente escolar. Este artigo traz algumas dessas produções. São apresentados três grupos de pesquisa, a saber: comportamento matemático e ensino de matemática; leitura; e comportamentos adequados (pró-sociais) e inadequados (agressões). Discutese como a localização geográfica (região Centro-Oeste) se torna um dos fatores relacionados à produção de conhecimento e, ainda, se apontam os aspectos facilitadores e limitantes do PSSP se localizar em tal região.

Palavras-chave: análise do comportamento; escola; matemática; leitura.

\begin{abstract}
Some contributions of behavior analysis on schools: Experiences from the Psychology Stricto Sensu Graduate Program (PSSP) at PUC Goiás. The Psychology Stricto Sensu Graduate Program (Programa de Pós-Graduação Stricto Sensu - PSSP) at the Pontifícia Universidade Católica de Goiás (PUC Goiás) was authorized in 1999. Behavior analysis is one of the fields of study in the program. Throughout 20 years it produced 115 dissertations and theses based on radical behaviorism. About $13 \%$ of the projects were conducted in a school environment. This study presents some of this production. We introduce three research groups, namely: mathematical behavior and teaching mathematics; reading; and adequate (prosocial) and inadequate (aggressive) behavior. We discuss how the geographical location (Centro-Oeste region of Brazil) is one of the factors related to knowledge production; and point out the facilitating and limiting aspects led by the program's location.
\end{abstract}

Keywords: behavior analysis; school; mathematics; reading.

\section{Resumen}

Contribuciones del análisis del comportamiento en la escuela: experiencias en el Programa de Postgrado Stricto Sensu en Psicología (PSSP) de la PUC Goiás. El Programa de Postgrado Stricto Sensu en Psicología (PSSP) de la Pontificia Universidad Católica de Goiás fue autorizado en 1999. El Análisis del Comportamiento es una de las áreas de concentración del programa. A lo largo de esos 20 años fueron producidos 115 teses y disertaciones basadas en el behaviorismo radical y aproximadamente $13 \%$ de los trabajos fueron conducidos en ambiente escolar. Este estudio presenta algunas de esas producciones. Son separados tres grupos de pesquisa, a saber: comportamiento matemático y enseñanza de matemáticas; lectura; y comportamientos adecuados (pro sociales) e inadecuados (agresiones) . Se discute como la ubicación geográfica (región Centro-Oeste) es uno de los factores relativos a la producción de conocimiento, apuntando aspectos facilitadores y limitantes del hecho de ese programa ubicarse en tal región.

Palabras clave: análisis del comportamiento; escuela; matemáticas; lectura. 
O Programa de Pós-Graduação Stricto Sensu em Psicologia (PSSP) da Pontifícia Universidade Católica de Goiás foi instituído em 1999, com o curso de Mestrado em Psicologia. Em 2006, o projeto do curso de Doutorado foi aprovado pela CAPES e, em 2007, foram abertas as primeiras vagas no processo seletivo de discentes para doutoramento. Desde a implantação do PSSP, a Análise do Comportamento, enquanto uma ciência do comportamento, foi contemplada em uma das áreas de concentração do PSSP.

Na época em que o PSSP foi instituído (1999), havia, no Brasil, ao menos outros quatro Programas de Pós-Graduação que contavam com áreas de concentração em Análise do Comportamento, sendo eles os da Universidade de São Paulo (USP), da Pontifícia Universidade Católica de São Paulo (PUC SP), da Universidade Federal do Pará (UFPA) e da Universidade de Brasília (UnB). A partir de 1999 diversos outros programas surgiram - Universidade Estadual de Londrina (UEL), Centro Universitário da Universidade Federal do Espírito Santo (UFES), Universidade Estadual Paulista (Unesp), Universidade Federal do Paraná (UFPR), Universidade do Vale do São Francisco (UNIVASF), assim como um segundo programa da Universidade Federal do Pará (UFPA). Além desses, outros programas brasileiros contam com analistas do comportamento, como a Universidade Federal de Santa Catarina (UFSC) e o Centro Universitário de Brasília (UniCEUB). Durante anos, o PSSP da PUC Goiás e o Mestrado em Psicologia (atualmente em Ciências do Comportamento) da UnB foram os únicos programas de pós-graduação localizados no Centro-Oeste.

A expansão de Programas de Pós-Graduação envolvendo pesquisadores da Análise do Comportamento ocorreu de modo diferenciado no Sul e no Sudeste em relação às demais regiões do país. Diversos fatores podem explicar tal diferença, como recursos financeiros, recursos humanos e outros elementos que podem ter atraído ou facilitado a manutenção de pesquisadores em Instituições de Ensino Superior (IES) localizadas nessas regiões. Apesar da diferenciação, o PSSP da PUC Goiás tem obtido sucesso no que se refere à produção de conhecimento. A produção docente qualificada na área, com índice crescente de defesas de dissertações de mestrado e teses de doutorado, é observada desde a implantação do programa. Sob a orientação dos analistas do comportamento que compõem o corpo docente do PSSP, essas publicações tratam de uma variedade de temas, investigados por meio de pesquisas básicas e aplicadas.

Para o desenvolvimento deste trabalho os autores solicitaram à Secretária do PSSP dados referentes às Teses e Dissertações defendidas desde a criação do programa. Em resposta ao pedido foram fornecidos dois arquivos nos quais constam informações sobre: resumo, palavras-chave, data de defesa de todas as teses e dissertações defendidas entre 2001 e 2018. Dois pesquisadores leram todos os resumos e selecionaram apenas os trabalhos que envolviam como base epistemológica a Análise do Comportamento. Em seguida os dois pesquisadores categorizaram os trabalhos como pertencentes à pesquisa básica, pesquisa aplicada e quais destes foram desenvolvidos em contexto escolar. Outros dois autores releram os trabalhos selecionados e as categorizações para dupla checagem da categorização.

Em relação à escolha dos trabalhos a serem apresentados a seguir não houve nenhum critério específico para escolha dos mesmos. Os autores optaram por apresentar trabalhos que já conheciam em detalhes (por envolver participação dos autores como pesquisadores, orientadores ou por participação em bancas avaliativas). Os autores também escolheram outros trabalhos que apesar de não serem produções do PSSP, permitiriam a construção de uma lógica racional das informações apresentadas. Para diferenciar os trabalhos produzidos no PSSP serão apresentados ao longo do texto seguidos de um asterisco.

Como resultado deste levantamento, notou-se um número relativamente estável de dissertações e teses pautadas em pesquisa básica desde a implantação do PSSP; por outro lado, percebe-se uma crescente produção, desde 2007, ano de implantação do doutorado, oriunda da pesquisa aplicada (ver Tabela 1). Em 19 anos, foram produzidos um total de 115 trabalhos e, destes, mais de $13 \%$ foram desenvolvidos em contexto escolar.

Tabela 1. Número de Teses e Dissertações no PSSP com Base em Análise do Comportamento por Tipo de Pesquisa Básica (PB) e Aplicada (PA) em outros Contextos e no Contexto Escolar por Ano e Total de Produção

\begin{tabular}{|c|c|c|c|c|c|}
\hline \multirow[b]{2}{*}{ Ano } & \multirow[b]{2}{*}{ PB } & \multicolumn{3}{|c|}{ Pesquisa Aplicada (PA) } & \multirow[b]{2}{*}{ Total de Produções } \\
\hline & & $\begin{array}{c}\text { Outros } \\
\text { Contextos }\end{array}$ & $\begin{array}{c}\text { Contexto } \\
\text { Escolar }\end{array}$ & Total PA & \\
\hline 2001 & 3 & 2 & 0 & 2 & 5 \\
\hline 2002 & 2 & 2 & 0 & 2 & 4 \\
\hline
\end{tabular}


Tabela 1. Continuação

\begin{tabular}{|c|c|c|c|c|c|}
\hline \multirow[b]{2}{*}{ Ano } & \multirow[b]{2}{*}{ PB } & \multicolumn{3}{|c|}{ Pesquisa Aplicada (PA) } & \multirow[b]{2}{*}{ Total de Produções } \\
\hline & & $\begin{array}{c}\text { Outros } \\
\text { Contextos }\end{array}$ & $\begin{array}{c}\text { Contexto } \\
\text { Escolar }\end{array}$ & Total PA & \\
\hline 2003 & 3 & 2 & 0 & 0 & 5 \\
\hline 2004 & 2 & 3 & 0 & 3 & 5 \\
\hline 2005 & 3 & 3 & 1 & 4 & 7 \\
\hline 2006 & 2 & 1 & 0 & 1 & 3 \\
\hline 2008 & 1 & 4 & 1 & 5 & 6 \\
\hline 2009 & 3 & 4 & 1 & 5 & 8 \\
\hline $2010^{* *}$ & 0 & $4(4 \mathrm{M})$ & $3(2 M 1 D)$ & 7 & 7 \\
\hline 2011 & 1 & 2 & 0 & 2 & 3 \\
\hline 2015 & 4 (3M 1D) & 3 & $2(2 M)$ & 5 & 9 \\
\hline 2016 & $3(1 M 2 D)$ & $3(3 \mathrm{D})$ & $1(1 \mathrm{M})$ & 4 & 7 \\
\hline 2017 & $3(3 \mathrm{M})$ & $6(3 M 3 D)$ & $1(1 \mathrm{M})$ & 7 & 10 \\
\hline 2018 & 1 (1D) & $2(2 D)$ & 0 & 2 & 3 \\
\hline
\end{tabular}

* $\mathrm{M}$ = Dissertações de Mestrado; $\mathrm{D}$ = Teses de Doutorado.

** Em 2010 foi o ano da primeira defesa de tese de doutorado no PSSP.

Dentre as dissertações e teses produzidas de 2007 até 2016, a pesquisa aplicada no contexto escolar apresenta-se como uma nova demanda e indica o interesse dos analistas do comportamento na investigação de fenômenos comportamentais que ocorrem nesse contexto. Assim, o objetivo do presente artigo é apresentar alguns achados de pesquisa relacionados ao contexto escolar produzidos no PSSP e discutir as dificuldades encontradas para a produção de conhecimento, uma vez que o PSSP não se encontra nas regiões Sul e Sudeste.

\section{Pesquisas envolvendo Matemática}

A análise do comportamento define os fenômenos psicológicos como eventos comportamentais e tem se esforçado para estudar como o comportamento é adquirido a partir da interação com o ambiente (Todorov, 2012). Nessa perspectiva, a aquisição de habilidades relacionadas à matemática pode também ser compreendida como comportamento.

A aquisição de repertórios matemáticos é considerada um desafio por diferentes profissionais da Educação. Frente a essa dificuldade, analistas do comportamento têm contribuído refinando conceitos e investigando metodologias e estratégias que favoreçam o ensino e a aprendizagem de habilidades matemáticas.
De forma geral, os trabalhos em análise do comportamento podem ser compreendidos em relação ao comportamento matemático e ao comportamento de ensinar matemática (Henklain, Carmo, \& Bender, 2017).

O comportamento matemático, segundo Carmo e Prado (2004), pode ser compreendido como um conceito referente às respostas emitidas por uma pessoa e os estímulos que a controlam, cuja relação é reforçada por uma comunidade verbal. Portanto, refere-se ao comportamento de quem aprende e é passível de ser analisado funcionalmente. Por sua vez, o comportamento de ensinar matemática, de acordo com Teixeira (2006), diz respeito ao comportamento de quem ensina, que também pode ser definido e analisado funcionalmente como uma relação entre o comportamento de quem aprende (e assim reforça o comportamento de quem ensina) e as respostas (estratégias e métodos) de quem ensina, facilitando a aquisição de habilidades matemáticas.

Dentre os vários comportamentos matemáticos de quem aprende e de quem ensina encontram-se os relacionados aos conceitos de proporção e fração. Uma fração pode ser definida como uma forma de expressão envolvendo quantidades de uma mesma natureza, que representa a relação entre as partes marcadas (tomadas) e o total de partes em que um inteiro 
(uma unidade) foi dividido. Relações entre diferentes frações são consideradas proporcionais se representarem magnitudes relativas entre elas, ou seja, se expressões matemáticas diferentes representarem uma mesma quantidade (Quintero, 1987; Skypek, 1984). Frações diferentes que representam o mesmo valor são chamadas de frações equivalentes (T. N. Carraher, Carraher, \& Schliemann, 1986). A resolução de problemas dessa natureza exige que o comportamento fique sob o controle de uma relação entre os estímulos apresentados na situação, estabelecendo, então, um responder relacional.

O ensino e a aprendizagem desse comportamento matemático relacional são considerados como desafios por diferentes profissionais da Educação e têm instigado a psicologia a investigar estratégias mais eficientes para sua aquisição (D. W. Carraher \& Schliemann, 1992; Santos, 1999; Spinillo, 1993). Segundo D. W. Carraher e Schliemann, (1992), a maior dificuldade demonstrada por alunos do Ensino Fundamental se trata da possibilidade de uma fração ser representada pela magnitude relativa entre o numerador e o denominador, sendo que nenhum de seus termos se refere a quantidades absolutas ou literais das expressões. Cabe destacar que a compreensão quanto à existência de proporcionalidade em diferentes formas de expressões matemáticas é requerida como forma de interação para a resolução de muitos problemas da vida diária, o que justifica o interesse em aprimorar seu ensino.

\section{Estudos Experimentais sobre o Ensino do Conceito de Frações}

O interesse em aplicar princípios do condicionamento operante para ensinar conceitos matemáticos é antigo na análise do comportamento (e.g., Iñesta, 1972). Recentemente Henklain et al. (2017) avaliaram a produção analítico-comportamental brasileira sobre comportamento matemático e de ensinar matemática no período compreendido entre os anos de 1970 a 2015. Nesse levantamento, foi demonstrado que a produção de trabalhos, considerando-se o comportamento matemático e o comportamento de ensinar matemática, desenvolvidos em forma de dissertações, teses ou trabalhos apresentados em eventos científicos, foi maior em Institutos de Ensino Superior (IES) das regiões Sul e Sudeste, nas quais foram identificados 114 trabalhos, de sete instituições diferentes. Nas regiões Norte e Nordeste, foram 47 produções de duas universidades; por fim, na região Centro-Oeste foram registrados apenas sete trabalhos, de duas instituições.

Alguns dos trabalhos realizados na região CentroOeste foram desenvolvidos a partir das contribuições de
Sidman e Tailby (1982), que demonstraram como treinar determinadas relações entre estímulos e estabelecer o que denominaram de classes de equivalência. Essa proposta despertou o interesse para pesquisas voltadas para a área escolar por exemplificar uma maneira de treinar relações diretas e indiretas entre estímulos arbitrários matemáticos e pode ser exemplificada pelo trabalho de Lynch e Cuvo (1995). Neste trabalho, Lynch e Cuvo (1995) ensinaram relações arbitrárias entre estímulos matemáticos apresentados de forma decimal (conjunto $A$, exemplo 0,5) e estímulos numéricos fracionários (conjuntos $B$, exemplo 1/2) e entre os estímulos fracionários dos conjuntos B e C (exemplo 1/2 e 2/4, respectivamente). Os resultados mostraram que os participantes demonstraram relações de simetria (BA e $C B$ ), transitividade (AC) e equivalência (CA), sem necessidade de treino direto.

A compreensão sobre as condições que viabilizam a formação de classes de estímulos arbitrários incentivou pesquisadores a investigar como ensinar e aprender o conceito de equivalência entre frações, uma vez que a aquisição deste conceito implica em formar classes de estímulos fracionários arbitrários. Algumas das pesquisas nesta área aconteceram na PUC Goiás. Esses estudos envolveram 14 discentes do curso de graduação em Psicologia e incluíram alunos de Programas de Iniciação Científica e voluntários de pesquisa. Como resultados dessas pesquisas, foram publicados três artigos em periódicos da psicologia e três publicações completas em anais de eventos científicos, além de quatro trabaIhos apresentados em congressos e encontros de psicologia (Santos, Cameschi, \& Hanna, 2009*; Santos, Silva, Santana, Montefusco, \& Oliveira, 1999*; Santos, Silva, \& Oliveira, 1999*; Santos, Simonassi, \& Magri, 2014*; Santos et al., 2015; Santos, Souza, \& Bay, 1997; ${ }^{*}$.

Nesses trabalhos, crianças que apresentaram dificuldade em solucionar problemas fracionários aplicados na prova de múltipla escolha (avaliação inicial) aprenderam a escolher frações numéricas correspondentes aos modelos pictóricos ou numéricos equivalentes em tarefa de pareamento com o modelo, de acordo com os critérios propostos por Sidman e Tailby (1982). Os treinos dessas relações condicionais aconteceram isoladamente ou em conjunto com variáveis como controle por regras, instruções programadas ou treino de cópia com resposta construída. Ao final, refizeram a prova de múltipla escolha, com problemas fracionários (avaliação final).

Os estudos realizados por Santos et al. (2009)*, Santos et al. (2014)* , e Santos et al. (2015)*, exemplificam os procedimentos e resultados observados nos demais estudos. Santos, Cameschi e Hanna (2009)*

Estudos de Psicologia, 24(2), abril a junho de 2019, 193-203 
compararam os desempenhos de participantes que passaram por treinos de relações condicionais com participantes que não fizeram esses treinos. Santos, Simonassi e Magri (2014* compararam quem passou por treinos de relações condicionais com quem fez os treinos dessas relações em conjunto com o treino de cópia e Santos et al. (2015)* compararam participantes que passaram por treino de relações condicionais com quem fez um programa de instrução programada sobre como resolver problemas fracionários e quem fez treino de relações condicionais intercalados com instruções programadas. De forma geral, os desempenhos observados na Avaliação Final (AF) da prova de múltipla escolha realizada após o treino das relações condicionais, foram maiores que os escores inicialmente demonstrados pelos participantes na Avaliação Inicial (AI) em todos os três experimentos.

Esses estudos tiveram como objetivo contribuir para a compreensão dos efeitos da formação de classes de equivalência no ensino de frações numa perspectiva da análise experimental do comportamento e buscaram mostrar como a aquisição de conceitos fracionários podem ocorrer em função da programação de contingências. Tomados em conjunto, os resultados indicam que o treino de relações condicionais pode, mesmo isoladamente, ser uma alternativa de estratégia para o ensino de frações, mas sua combinação com outros procedimentos ainda necessita de mais investigações.

\section{Estudos Experimentais sobre o Ensino do Comporta- mento de Leitura}

Na PUC Goiás, a primeira intervenção realizada para o ensino de leitura e escrita ocorreu em 1974. Os professores Lorismario Ernesto Simonassi e Márcio de Queiroz Barreto - da então Universidade Católica de Goiás (UCG), alfabetizaram um adolescente de 11 anos no Centro de Orientação Psicológica (COPSI) da universidade. O menino tinha histórico de fracasso escolar com o uso dos métodos tradicionais de ensino. O procedimento utilizado para essa intervenção foi o mesmo proposto por Iñesta (1972) dois anos antes e mostrou-se eficaz para o ensino de leitura.

Os resultados das pesquisas realizadas pelo PSSP no contexto escolar têm sugerido que os procedimentos de ensino utilizados são eficazes em casos de queixa de atraso no desenvolvimento da leitura. Para o ensino desse repertório tem-se usado: procedimento de escolha de acordo com o modelo associado ao esvanecimento gradual de pistas discriminativas (fading out); correção dos erros; apresentação de consequências não aversivas frente ao erro e de consequências reforçadoras em situações de acerto; controle do número de repetições da tarefa; uso de reforçamento social para consequenciar os comportamentos emitidos pelos participantes; procedimento de aprendizagem sem erro; aprendizagem por exclusão; dentre outros.

A aprendizagem de leitura tem sido investigada no PSSP com diversas populações, como crianças com diagnóstico de autismo (e.g. Bernardes \& Simonassi, 2016*) e crianças (e. g. Silva, 2012; Tizo \& Simonassi, $2008^{*}$ ) e adolescentes com desenvolvimento típico (e.g. Monteiro, 2018*).

Magalhães (2019)*, por exemplo, conduziu um estudo com duas crianças com características do Transtorno do Espectro Autista (TEA), que apresentavam controle restrito de estímulos. Elas foram treinadas utilizando os procedimentos descritos anteriormente e aprenderam a ler. Monteiro (2018)* , por sua vez, fez uso de procedimentos para viabilizar uma aprendizagem de leitura sem erros. Teve como participante um adolescente de 14 anos, com queixas de atraso no desenvolvimento de leitura e utilizou um programa de correções, realizadas sistematicamente, de cada unidade mínima. Evitou-se o excesso de repetições de uma mesma palavra durante o treino na ocorrência de erros, buscando diminuir eventual aversão à tarefa. Observou-se que o jovem passou a discriminar os estímulos verbais textuais apresentados ao longo da pesquisa a partir do rearranjo das contingências de ensino.

O estudo de Bernardes e Simonassi (2016)* replicou sistematicamente o programa de leitura proposto por Iñesta (1972). Participaram do estudo dois adolescentes, de 10 e 11 anos, com características do Transtorno do Espectro Autista (TEA), que ainda não liam. Verificou-se o estabelecimento e a manutenção do repertório de leitura, assim como a generalização de estímulos textuais, o que é uma característica desejável ao se instalar comportamento de leitura.

O estudo de Silva (2012)* foi realizado com quatro crianças, de 7 a 9 anos, da zona rural do estado de Goiás. O procedimento foi escolhido de acordo com o modelo baseado na discriminação sem erro, de Terrace (1963). Procedimentos de esvanecimento foram utilizados, tendo a aquisição de leitura ocorrido com todos os quatro participantes, que voltaram a se matricular em uma escola regular da região. Observações adicionais mostraram que uma das crianças passou a ser líder de um grupo de leitura da escola e que a aquisição da leitura foi pré-requisito para sua inclusão social. 
As pesquisas desenvolvidas pelo PSSP sobre o comportamento de ler têm contribuído para o desenvolvimento de programas de ensino que permitem a ocorrência da aprendizagem de forma mais rápida do que com o uso dos métodos tradicionais de ensino.

\section{Uma experiência junto ao Programa de Pesquisas para o Sistema Único de Saúde (PPSUS)}

Outra inserção do PSSP utilizando a base epistemológica do Behaviorismo Radical foi fomentada pelo Edital FAPEG/PPSUS 2013: Programa de Pesquisa para o SUS: Gestão compartilhada em Saúde - PPSUS/GO. O objetivo do edital foi apoiar projetos de pesquisa capazes de promover a formação de recursos humanos qualificados e melhorar a atenção à saúde no contexto do Sistema Único de Saúde (SUS). O projeto intitulado "Programa Saúde na Escola (PSE): avaliação dos efeitos da linha de ação e promoção das violências"1 foi contemplado. Parte do estudo foi identificar e analisar os instrumentos de avaliação, tipos e eficácia de intervenções já realizadas por esse Programa na cidade de Goiânia, de acordo com os princípios da Análise do Comportamento.

O projeto resultou em diferentes produções: um capítulo de livro (Neves et al, no prelo*), um artigo submetido (Neves et al., 2019*), um artigo publicado (Melo Jr, Souza, Baia, \& Neves, 2014*), uma orientação de mestrado e sete orientações de iniciação científica, além de apresentações em congressos. O conhecimento gerado permitiu compreender, em termos de contingências e metacontingências (Glenn et al., 2016), a regulamentação do PSE. O atendimento de metas pactuadas (a quantidade de estudantes atendidos em diferentes ações) produziu a liberação de recursos. Nesse caso, havia uma metacontingência programada. Cada agente envolvido - enfermeiros, médicos, agentes de saúde, professores, cirurgiões dentistas - atuava em sua respectiva profissão (esta seria a contingência comportamental entrelaçada - CCE), gerando um efeito no ambiente, chamado de Produto Agregado - número de estudantes atendidos -, que liberava recursos financeiros como consequências culturais (CC).

Em outra frente de trabalho, foi avaliada a estratégia da escola para registro de violências ocorridas no local (Neves et al., no prelo*). Nesse sentido, detectou-se que o principal instrumento para registro dos episódios violentos era o Livro de Ocorrências, um caderno no qual eram registrados diversos episódios ocorridos na escola, entre eles o descumprimento de normas escolares como não usar uniforme. Das 329 ocorrências identificadas, 181 envolviam comportamentos violentos e 148 não violentos. A análise do Livro de Ocorrências permitiu detectar que o modo como os eventos foram registrados impede uma compreensão adequada de quem eram os atores envolvidos, em qual contexto a situação ocorreu e qual foi o desfecho do episódio.

Também foram avaliadas as interações entre professores e alunos de uma escola em Goiânia que apresentava alto índice de violência (Neves et al., 2019*). Foram realizados registros das interações durante as aulas. Após uma avaliação preliminar foram construídos dois grupos, o grupo Disciplinados foi composto pelos alunos com o maior índice de comportamentos adequados. Já o grupo Indisciplinados foi composto por alunos com maior índice de comportamento inadequados. Para mensuração dos comportamentos adequados e inadequados os autores criaram categorias e analisaram a frequência dos comportamentos emitidos por cada aluno. Os dados dos comportamentos dos estudantes foram analisados individualmente e em grupo. Após a avaliação preliminar, Neves et al. $\left(2019^{*}\right)$ analisaram os comportamentos dos alunos registrando eventos antecedentes, respostas e consequências. Os resultados indicam que foram mais frequentes os comportamentos apropriados para os alunos do grupo Disciplinados. Já os comportamentos inapropriados não apresentaram diferença entre os dois grupos. Também foi possível notar que os professores raramente apresentavam consequência para os comportamentos dos alunos, a despeito de serem apropriados ou inapropriados.

A última parte do projeto aprovado no PPSUS envolveu uma intervenção em uma escola de Rio Verde, estado de Goiás; os resultados deste estudo ainda não haviam sido publicados e serão apresentados a seguir. O objetivo foi avaliar se o Jogo do Bom-Comportamento (JBC) - ou Good Behavior Game, em inglês - poderia ser conduzido em sala de aula por apenas uma professora e com baixo custo financeiro. Em geral, o JBC envolve a distribuição de alunos em equipes, o estabelecimento de regras - que descrevem quais comportamentos devem ocorrer e quais não devem -, além da premiação para as equipes que atingirem um critério especificado, como uma dada frequência de comportamentos-alvo (Pennington \& Mccomas, 2017). Como a intervenção era parte do projeto de pesquisa financiado pelo PPSUS2013 , os pesquisadores optaram por tentar contribuir

Estudos de Psicologia, 24(2), abril a junho de 2019, 193-203 
com informações sobre o uso do JBC de modo adaptado à realidade brasileira. Esse jogo é uma das estratégias adotadas pelo Ministério da Saúde sob o título de Programa Elos - Construindo Coletividades. Segundo Biglan (2015), o JBC tem sido efetivo para prevenção de uso e abuso de substâncias em crianças, o que justifica sua condução por parte do Ministério da Saúde.

Schneider et al. (2016) relataram a percepção de pedagogos, administradores escolares e aplicadores do JBC em um estudo piloto desenvolvido em quatro escolas brasileiras. Os autores aplicaram questionários estruturados e entrevistaram os profissionais envolvidos no projeto. Os resultados indicam que os participantes consideraram que o JBC é fácil de ser implementado em sala de aula e produz bons resultados, já que reduziu a frequência de comportamentos inapropriados e aumentou a participação de crianças em atividades. Porém, os profissionais destacaram que o jogo deve ser mais bem adaptado à realidade cultural brasileira. Outro problema são os recursos oferecidos para implantação do jogo, já que, segundo os professores, não houve disponibilidade adequada.

No caso da pesquisa sobre o JBC realizada envolvendo membros do PSSP da PUC Goiás, a professora/ aplicadora tinha encontros semanais com um dos pesquisadores para discutir as etapas e a leitura de textos referente ao JBC. Além disso, os pesquisadores forneceram matérias de baixo-custo, como material escolar para ser utilizado como premiação. Desse modo, os custos com os prêmios foram inferiores a 10 reais por semana. A pesquisa foi conduzida durante as aulas de duas escolas estaduais (Sexto Ano - Escola 1 e Sétimo Ano Escola 2) de Rio Verde, em Goiás. Os comportamentos-alvo escolhidos foram: (1) erguer a mão e aguardar autorização da professora para falar; (2) permanecer sentado em sua cadeira durante a explicação e atividades - exceto para ir ao banheiro ou descartar material no lixo; (3) realizar as tarefas solicitadas pela professora durante a aula ou em casa (nesse caso, o registro dependia de exibir a tarefa realizada nos minutos iniciais da aula seguinte ao pedido de tarefa domiciliar); (4) proferir elogios aos comportamentos de ajuda e gentileza dos colegas de classe - aqui, o aluno solicitava autorização e escrevia na placa o comportamento e o nome do colega.

Um dos passos a ser seguido é a observação da linha de base, uma etapa em que os comportamentos-alvo são registrados sem a interferência dos pesquisadores. Os pesquisadores da PSSP da PUC Goiás optaram por uma intervenção na qual a linha de base (LB) foi realizada em períodos distintos para turmas da mesma escola. Assim, os pesquisadores consideraram que poderiam avaliar se possíveis alterações na frequência dos comportamentos-alvo se dariam pela implementação do JBC ou por influência de qualquer outra variável estranha desconhecida. Assim, a Turma 1 (Escola 1) e a Turma 3 (Escola 2) permaneceram na LB por sete dias úteis consecutivos. Já a Turma 2 (Escola 1) e a Turma 4 (Escola 2) permaneceram na LB por 19 dias - paralisações dos professores resultaram no aumento de dias de linha de base.

A Figura 1 apresenta os resultados da LB para cada turma. Os dados apresentam a frequência conjunta de todos os comportamentos-alvo. Cada ponto na figura representa a média de comportamentos. A despeito da duração da LB (sete ou 19 dias), os comportamentos-alvo não aumentaram sua frequência média ao longo dos dias. Portanto, não se pode atribuir as mudanças na frequência média, durante a intervenção, a variáveis estranhas.

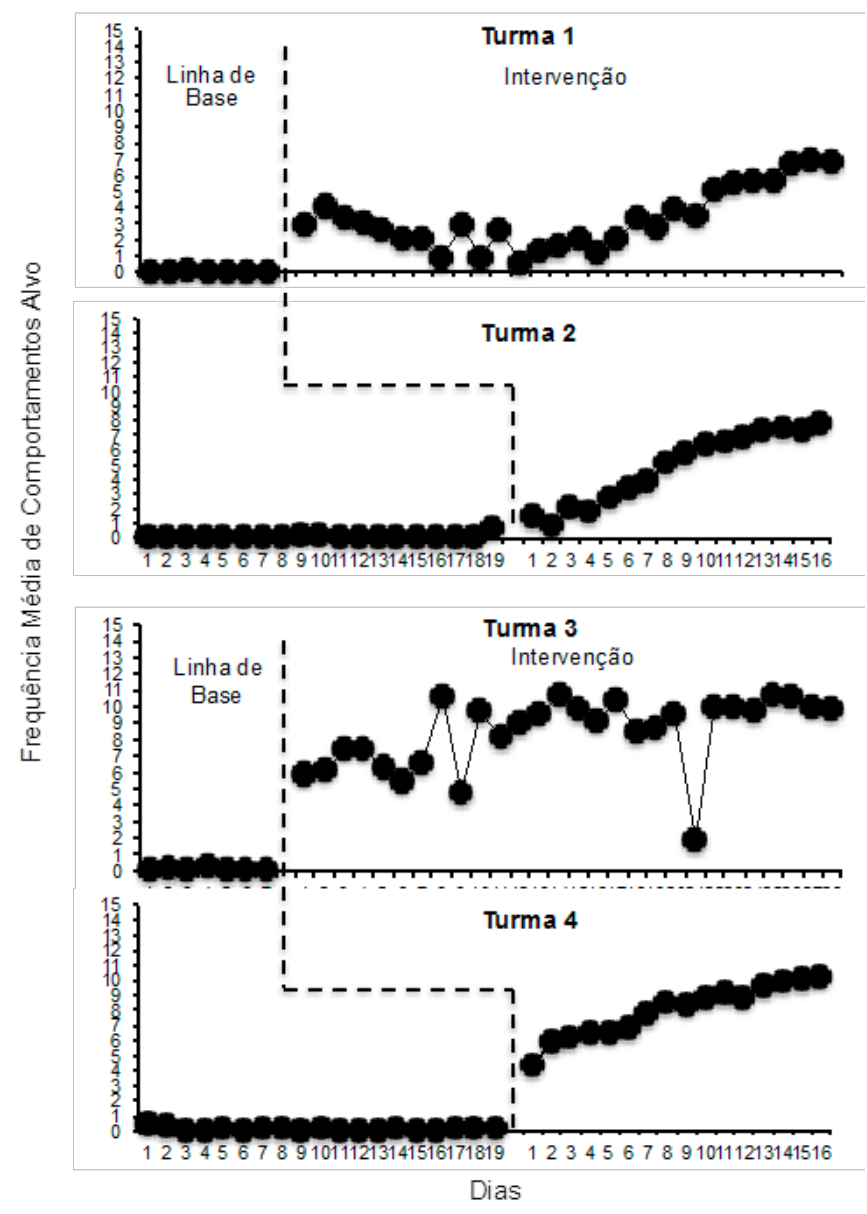

Figura 1. Frequência Média de Comportamentos-Alvo para Turma 1 e Turma 2 - Escola 1, Sexto Ano (Painéis Superiores) e Turma 3 e Turma 4 - Escola 2 (Painéis Inferiores) Referente à Pesquisa sobre O JBC. Linhas Tracejadas Separam a Linha de Base da Fase de Intervenção. Cada Ponto Representa a Frequência Media (I.E, Soma do Total de Comportamentos Dividido Total de Equipes). 
A intervenção teve início com o estabelecimento de regras. A cada semana um comportamento era definido como alvo, ou seja, um novo comportamento era elegível para pontuação. Para ressaltar quais eram os comportamentos-alvo que produziam pontos foi afixado um cartaz, no quadro-negro, com a descrição de cada um deles. Também foi informado, pela professora/aplicadora, que a cada ocorrência do comportamento-alvo de cada membro da equipe um ponto seria adicionado no placar. Assim, não havia registro individual de quem se comportava (programação interdependente). Por fim, foi explicado aos alunos sobre a premiação.

Os resultados da Fase Intervenção podem ser observados na Figura 1, painéis superiores (Turma 1 e Turma 2) e painéis inferiores (Turma 3 e Turma 4). $\mathrm{O}$ aumento da frequência média de comportamentos-alvo foi gradual. Algumas oscilações nessa frequência ocorreram durante a pesquisa - Turma 1, entre os dias 8 e 12, Turma 3, entre os dias 7, 8 e 21 . Tais oscilações estão ligadas a eventos ocorridos na escola. Por exemplo, no caso da Turma 8, a quantidade de alunos por grupo variou, pois o transporte da zona rural para a escola não funcionou nesses dias. Já para a Turma 3 , as aulas foram interrompidas para "conselhos de classe", os alunos foram liberados no horário do intervalo de recreio, e, por isso, houve redução no tempo de aula. De modo geral, os resultados do uso do JBC foram efetivos no estabelecimento de comportamentos cooperativos e de respeito entre os alunos participantes. A professora/aplicadora descreveu que achou fácil aplicar o JBC em suas aulas cotidianas, destacando, inclusive, que os recursos materiais utilizados eram de baixo custo e poderiam ser fornecidos por ela ou pela própria escola. Disse, ainda, que sem os encontros semanais com os pesquisadores a tarefa não poderia ser executada.

A condução do projeto de pesquisa relatado nesta seção só foi possível graças ao financiamento da Fundação de Amparo à Pesquisa de Goiás (FAPEG), em parceria com o Ministério da Saúde, por meio do PPSUS. A capacitação de mão de obra - como os alunos de graduação envolvidos - só foi conseguida graças ao apoio institucional das universidades envolvidas, a PUC Goiás e a UniRV, que forneceram bolsas de Iniciação Científica para alguns alunos que participaram deste projeto. A discente de Mestrado do PSSP também recebeu bolsa da CAPES, o que permitiu que se dedicasse ao PSSP e ao projeto de pesquisa.

\section{Discussão}

As produções do PSSP com base em Análise do Comportamento podem ser discutidas em dois aspectos: (1) a contribuição para a literatura sobre os assuntos investigados e (2) a importância do PSSP na região CentroOeste. As pesquisas realizadas no PSSP contribuíram, por exemplo, para mostrar a robustez de procedimentos da Análise do Comportamento para ensino de leitura com diversas populações. Os resultados, de maneira geral, sugerem que a associação de procedimentos amplamente utilizados pela Análise do Comportamento (e.g., discriminação sem erro de Terrace, 1963; fading out; a não utilização de consequências aversivas; uso de reforçamento social, dentre outros) é eficaz para o ensino de repertórios novos, sendo uma alternativa frente às formas tradicionais de ensino de leitura.

No que diz respeito ao ensino de comportamento matemático, as pesquisas realizadas no PSSP contribuem para literatura da área ao investigar variáveis que poderiam aumentar a compreensão sobre como o professor poderia programar atividades para o ensino do conceito de equivalência entre frações numa perspectiva da análise experimental do comportamento e, assim, facilitar a aprendizagem do aluno. Os trabaIhos sobre matemática desenvolvidos no PSSP corroboraram dados da literatura que indicam dificuldades para o ensino e aquisição de conceitos fracionários (D. W. Carraher \& Schliemann, 1992; Santos, 1999). De forma geral, estes estudos investigaram como o paradigma de equivalência de estímulos, de forma isolada ou em combinação com outros procedimentos, pode ser uma alternativa promissora como estratégia pedagógica para o ensino do conceito de equivalência entre frações, e corroboraram com resultados de trabalhos anteriores como o desenvolvido por Linch e Cuvo (1995).

Em relação aos trabalhos fomentados pelo Edital FAPEG/PPSUS 2013 os resultados obtidos são de modo geral similares àqueles encontrados na literatura. No estudo de Neves et al. (no prelo) foi observado que consequências - por exemplo "enviado para Diretoria" foi o componente comportamental com maior frequência nos registros. Esse resultado é similar ao apontado em outros estudos, que sinalizam que "convocar os pais" é a medida mais adotada para violência escolar. Já as análises da interação professor-aluno investigadas em Neves et al. (2019) revelaram que a consequência mais comum para comportamentos inapropriados era de caráter aversivo. Os comportamentos apropriados 
em geral não eram consequenciados - os professores ignoravam sua ocorrência. Esses resultados são similares aos observados por Bolsoni-Silva, Mariano, Loureiro e Bonaccorsi (2013).

Por fim, em relação à pesquisa sobre o JBC os resultados sobre a ocorrência de comportamentos desejados - aqueles estabelecidos no jogo - demonstra que os mesmos aumentaram de frequência ao longo das semanas um resultado típico da literatura (ver Pennington \& Mccomas, 2017). Durante as reuniões de orientação a professora declarou diversas vezes que a aplicação do JBC era de fácil execução e que os custos de premiação eram baixos. Esse resultado sugere que as alterações feitas no estudo sobre o JBC foram efetivas em adaptá-lo a realidade brasileira.

No que se refere à importância do PSSP na região Centro-Oeste, O PSSP PUC Goiás foi o segundo Programa de Pós-Graduação Stricto Sensu em Psicologia a ser instituído na região Centro-Oeste. Atualmente, há outras sete instituições com programas de Pós-Graduação em Psicologia. Das oito IES com programas de pós-graduação no Centro-Oeste, apenas três possuem curso de mestrado e doutorado (UnB, UCDB e PUC Goiás). Esses dados são contrastantes com os valores nacionais. São oferecidos 26 cursos de Mestrado Acadêmico, 14 de Mestrado Profissionalizante e 60 de Mestrado e Doutorado em todo o Brasil. Na Região Sudeste, são cinco cursos de Mestrado Acadêmico, seis Mestrados Profissionalizantes e 27 programas de Mestrado e Doutorado.

A quantidade de programas de Pós-Graduação pode ser uma das variáveis que explicam a diferença no quantitativo de produtos como artigos, teses e dissertações. Por exemplo, na revisão de trabalhos sobre comportamento matemático realizada por Carmo e Bender (2017) identificou-se que as regiões Sul e Sudeste foram responsáveis por 114 produtos; já a região Centro-Oeste apresentou sete produções. Outra forma de expor a discrepância entre as regiões é por meio dos laboratórios que participam do Instituto Nacional de Ciência e Tecnologia Sobre Comportamento Cognição e Ensino (INCT-ECCE). O INCT-ECCE é o maior instituto nacional e reúne pesquisadores que atuam em pesquisas sobre Comportamento e Cognição. Apesar de haver pesquisadores do Centro-Oeste que investigam leitura (um dos principais temas investigados pelo INCT-ECCE) desde a década de 1970, apenas dois laboratórios fazem parte do INCT. Dos 14 laboratórios integrantes, 11 encontram-se na região Sudeste (UFSCar, USP, Unesp e UFMG e CEI).
Outra variável que pode ser destacada como desafios enfrentados para a produção de conhecimento na região Centro-Oeste são os recursos financeiros, que permitem tanto a aquisição de equipamentos e custeio para a realização de pesquisas quanto o pagamento de recursos humanos. Assim, mais recursos produzem melhores condições de trabalho que, por sua vez, geram mais possibilidade de produção de conhecimento. Possivelmente, tais fatores (condições de trabalho e índice de produção) atraem mais pesquisadores. A utilização de recursos de editais de fomento é uma oportunidade para atrair recursos financeiros para a condução de pesquisas em Goiás. Oscilações políticas no Governo do Estado e, por conseguinte, na FAPEG, ora dificultam, ora facilitam a condução de projetos de pesquisa. Os recursos em editais nacionais são muitas vezes de difícil obtenção, dada a disparidade entre as possibilidades de recursos entre os estados. Nesse sentido, pesquisadores de São Paulo encontram mais oportunidades para produzir, o que aumenta a possibilidade de serem contemplados com mais recursos nacionais.

Assim, a inserção de analistas do comportamento conduzindo intervenções em escolas de Goiás dependeu dos pesquisadores envolvidos serem agraciados com recursos financeiros de agências de fomento. Por um lado, o fato de uma das pesquisadoras atuar como orientadora de Mestrado e Doutorado pode ter aumentado a chance de contemplação no edital. Por outro, os recursos obtidos permitiram a capacitação de alunos de graduação, sendo que dois deles hoje são mestres pelo PSSP. Assim, os recursos financeiros serviram tanto para fomentar a pesquisa quanto para atrair novos pesquisadores (isto é, discentes) para o PSSP, da PUC Goiás. Nesse sentido, o PSSP tem sido fundamental na produção de conhecimento em Análise do Comportamento no estado de Goiás e na região Centro-Oeste. Na ausência de editais de fomento, a produção de conhecimento fica praticamente restrita aos programas de Pós-Graduação.

Outro ponto que revela a importância do PSSP para a região é que muitos dos egressos do PSSP hoje são professores em IES do Centro-Oeste. Aqueles que não são egressos mantêm relações de parceria para o desenvolvimento de pesquisas (como é o caso do segundo e sétimo autores deste trabalho). O PSSP, além de fornecer docentes para outras IES, também recebe egressos de outras instituições, como a UnB. Desse modo, o PSSP também é importante na fixação de doutores no estado de Goiás e na região Centro-Oeste e, logo, atua como um facilitador para a produção de conhecimento. 


\section{Referências}

Bernardes, L. V., \& Simonassi, L. E. (2016). Programa de leitura para pessoas com diagnóstico de autismo. Brasília: Instituto Walden4. Recuperado de https://www.walden4.com.br/livrosw4/pdf/bernardes_ simonassi_2016_iw4.pdf

Biglan, A. (2015). The nurture effect: How the Science of Human Behavior can improve our lives \& our world. Oakland: New Harbinger Publications.

Bolsoni-Silva, A. T., Mariano, M. L., Loureiro, S. R., \& Bonaccorsi, C. (2013). Contexto escolar: práticas educativas do professor, comportamento e habilidades sociais infantis. Psicologia Escolar e Educacional, 17(2), 259-269. doi: 10.1590/S1413-85572013000200008

Carmo, J. S., \& Prado, P. S. T. (2004). Análise do comportamento e psicologia da educação matemática: algumas aproximações. In M.M. C. Hübner \& M. Marinotti (Orgs.), Análise do comportamento para a educação: contribuições recentes (pp. 115-136). Santo André: Esetec.

Carraher, T. N., Carraher, D.W., \& Schliemann, A.D. (1986). Proporcionalidade na educação científica e matemática: desenvolvimento cognitivo e aprendizagem. Revista Brasileira de Estudos Pedagógicos, 67, 586-602.

Carraher, D. W., \& Schliemann, A. D. (1992). A compreensão de frações como magnitudes relativas. Psicologia: Teoria e Pesquisa, 8, 67-78. Recuperado de http://periodicos.unb.br/index.php/revistaptp/article/ view/17124

Glenn, S. S., Malott, M. E., Andery, M. A. P. A., Benvenuti, M., Houmanfar, R., Sandaker, I, ... Vasconcelos, L. A. (2016). Toward consistent terminology in a behaviorist approach to cultural analysis. Behavior and Social Issues, 25, 11-27. doi: 10.5210/bsi.v.25i0.6634

Henklain, M. H. O., Carmo, J. S., \& Haydu, V. B. (2017). Produção analíticocomportamental brasileira sobre comportamento matemático e de ensinar matemática: dados de 1970 a 2015. Temas em Psicologia, 25(3), 1453-1466. doi: 10.9788/TP2017.3-24

Iñesta, E. R. (1972). Técnicas de modificación de conducta: su aplicación al retardo en el desarrollo. México: Editorial Trillas S. A.

Lynch, D. C., \& Cuvo, A. J. (1995). Stimulus equivalence instruction of fraction-decimal relations. Journal of Applied Behavior Analysis, 28, 115-126. doi: 10.1901/jaba.1995.28-115

Magalhães, R. B. P. S. (2019). Controle restrito na leitura: avaliação de crianças com transtorno do espectro autista (Dissertação de Mestrado não publicada). Pontifícia Universidade Católica de Goiás, Goiânia.

Melo Jr., I. F., Souza, A. C. G., Baia, F. H., \& Neves, S. M. M. (2014). Obesidade e as intervenções comportamentais: análises de macrocontingências e metacontingências. Fragmentos de Cultura, 24, 3-13. doi: 10.18224/frag.v24i0.3560

Monteiro, S. P. (2018). Procedimentos corretivos à aprendizagem sem erro: o caso de um adolescente com fracasso sistemático em leitura (Tese de doutorado, Pontifícia Universidade Católica de Goiás, Goiânia). Recuperado de http://tede2.pucgoias.edu.br:8080/handle/tede/4009

Neves, S. M. M., Bomfim, F. C., Todorov, J. C., Baia, F. H., Souza, A. C. G., \& Melo Júnior, I. F. (no prelo). Análise Funcional de comportamentos violentos registrados em Livro de Ocorrências. Comportamento em Foco.

Neves, S. M. M, Todorov, J. C., Cerquim, F. S., Baia, F. H., Souza, A. C. G., \& Melo Júnior, I. F. (2019). Avaliação funcional dos comportamentos apropriados e inapropriados na relação professor aluno. Manuscrito submetido para publicação.

Pennington, B., \& McComas, J. J. (2017). Effects of Good Behavior Game across classroom contexts. Journal of Applied Behavior Analysis, 50(1), 176-180. doi: 10.1002/jaba.357

Quintero, A. H.(1987). Helping children understand ratios. Arithmetic Teacher 34(99), 17-21. Recuperado de https://www.jstor.org/stable/41194224
Santos, A. C.G. (1999). Equivalência de frações: como anda a compreensão dos alunos? Estudos: Revista da Universidade Católica de Goiás, 26(2), 233-249.

Santos, A. C. G., Cameschi, C., \& Hanna, E. S. (2009). Ensino de frações baseado no paradigma de equivalência de estímulos. Revista Brasileira de Análise do Comportamento, 5, 19-41. doi: 10.18542/rebac.v5i1.706

Santos, A. C. G., Silva, C. H. N. E., Santana, L. A. M., Montefusco, M. R., \& Oliveira, A. M. P. (1999). Efeito do controle instrucional e do treino de relações numérico-numérico em tarefas de pareamento com o modelo na aquisição do conceito de proporção. In Sociedade Brasileira de Psicologia (Org.), XXIX Reunião Anual de Psicologia (pp. 85-86). Campinas: Autor.

Santos, A. C. G., Silva, C.H. N.E., \& Oliveira, E. L. (1999). Aprendizagem no conceito de proporção e o paradigma de equivalência de estímulos: efeito do treino numérico-numérico sobre o desempenho generalizado. In Sociedade Brasileira de Psicologia (Org.), XXIX Reunião Anual de Psicologia (pp. 83-83). Campinas: Autor.

Santos, A. C. G., Simonassi, L. E., \& Magri, M. R. (2014). Efeito do treino de composição (cópia) naaprendizagem do conceito de proporção.Psicologia: Teoria e Pesquisa, 30(4), 459-469. Recuperado de http://www.scielo.br/ scielo.php?script=sci_arttext\&pid=S0102-37722014000400011\&lng=p t\&nrm=iso

Santos, A. C. G., Simonassi, L. E., Queiroz, R. F., Pacheco, F. B. P., Santana M. M., Magri, M. R., \& Melo, G.H.H. (2015). Equivalência de estímulos e instrução programada: formação de classes com estímulos fracionários. Itinerarius Reflectionis, 11(1). doi: 10.5216/rir.v1111.33341

Santos, A. C. G., Souza, A. V., \& Bay, C. K. (1997). Aprendizagem do conceito de proporção: interação entre equivalência de estímulos e instruções. [Resumo]. In Sociedade Brasileira de Psicologia (Org.), Anais da XXVII Reunião Anual da Sociedade Brasileira de Psicologia. Campinas: Autor. Recuperado de https://www.sbponline.org.br/anais-e-resumos

Schneider, D. R., Pereira, A. P. D., Cruz, J. I., Strelow, M., Chan, G., Kurki, A., \& Sanchez, Z. M. (2016). Evaluation of the implementation of a preventive program for children in Brazilian schools. Psicologia: Ciência e Profissão, 36(3), 508-519. doi: 10.1590/1982-3703000592016

Sidman, M., \& Tailby, W. (1982). Conditional discrimination vs. matching to sample: an expansion of the testing paradigm. Journal of the Experimental Analysis of Behavior, 37, 5-22. doi: 10.1901/jeab.1982.37-5

Silva, D. N. (2012). Programa de leitura e sua aplicação ao atraso no desenvolvimento (Dissertação de Mestrado, Pontifícia Universidade Católica de Goiás, Goiânia). Recuperado de http://tede2.pucgoias.edu. br:8080/bitstream/tede/1800/1/Denise\%20Neves\%20da\%20Silva.pdf

Skypek, D. H. B. (1984). Special characteristics of rational numbers. Arithmetic Teacher, 31(6), 10-12. Recuperado de https://www.jstor. org/stable/41191001

Spinillo, A.G. (1993). As relações de primeira-ordem em tarefas de proporção: uma outra explicação quanto às dificuldades das crianças. Psicologia: Teoria e Pesquisa, 9, 349-364. Recuperado de http://www.scielo.br/pdf/ $\mathrm{prc} / \mathrm{v} 15 \mathrm{n} 3 / \mathrm{a} 03 \mathrm{v} 15 \mathrm{n} 3 . \mathrm{pdf}$

Teixeira, A. M. S. (2006). Análise de contingências em programação de ensino infantil: Liberdade e efetividade na educação. Santo André: Esetec.

Terrace, H. S. (1963). Discrimination learning with and without "errors". Journal of the Experimental Analysis of Behavior, 1, 1-27. doi: 10.1901/ jeab.1963.6-1

Tizo, M., \& Simonassi, L. E. (2008). Aquisição de vocabulário: efeito de estímulos novos no controle de respostas. Revista Brasileira de Análise do Comportamento, 4(2), 205-217. doi: 10.18542/rebac. v4i2.851

Todorov, J. C. (2012). Sobre uma definição de comportamento. Revista Perspectivas, 3(1), 32-37. doi: 10.18761/perspectivas.v3i1.79

Estudos de Psicologia, 24(2), abril a junho de 2019, 193-203 
1. O projeto de pesquisa foi aprovado pelo Comitê de Ética em Pesquisas com o CAAE: 34169814.5.0000.0037.

Sonia Maria Mello Neves, Doutora em Psicologia Experimental pela University College of North Wales - UCNW, Bangor - UK, Pós-doutora pela Universidade de Brasília - UnB, é Professora Titular da Pontifícia Universidade Católica de Goiás - PUC-Goiás. Endereço para correspondência: Rua J 51 quadra 136 lote 31, Setor Jaó. Goiânia - Goiás. CEP 74.6741-70. Telefone: (62) 999792437. Email: soniamelloneves@gmail.com

Fábio Henrique Baia, Doutor em Ciências do Comportamento pela Universidade de Brasília - UnB, Pós-Doutorando pela Universidade de São Paulo - USP, é Professor Titular da Universidade de Rio Verde - UniRV.Email: fabio@unirv.edu.br

Antônio Carlos Godinho Santos, Doutor em Processos Comportamentais Básicos pela Pontifícia Universidade Católica de Goiás - PUC-Goiás, é Professor Adjunto da Pontifícia Universidade Católica de Goiás - PUC-Goiás.

Email: acgodinhos@gmail.com

Gabriela Sebastiana Ciriaco, Graduanda em Psicologia pela Pontifícia Universidade Católica de Goiás - PUC-Goiás. Email: Gabrielaciriaco83@gmail.com

Lorismario Ernesto Simonassi, Doutor em Psicologia Experimental pela Universidade de São Paulo - USP, é Professor Titular da Pontifícia Universidade Católica de Goiás - PUC-Goiás. Email: lorismario@gmail.com

Nagi Hanna Salm Costa, Doutora em Ciências do Comportamento pela Universidade de Brasília - UnB, é Professora Convidada da Pontifícia Universidade Católica de Goiás - PUC-Goiás. Email: nagi.hanna@hotmail.com

Rogério Guaita dos Santos Baia, Graduado em Enfermagem pela Universidade de Rio Verde - UniRV. Email: rogerguaitabaia@gmail.com

Recebido em 31.mai.19 Revisado em 22.set.19 Aceito em 12.nov.19 